\title{
Spectral weight transfer in the integer quantum Hall effect and its consequences
}

\author{
CHENGGANG ZHOU ${ }^{1 *}$ AND MONA BERCIU² \\ ${ }^{1}$ Center for Nanophase Materials Sciences, Oak Ridge National Laboratory, PO Box 2008, MS 6493, Oak Ridge, Tennessee 37831-6493, USA \\ ${ }^{2}$ Department of Physics and Astronomy, University of British Columbia, Vancouver B.C. V6T 1Z1, Canada \\ *e-mail: zhouc@ornl.gov
}

Published online: 2 December 2007; doi:10.1038/nphys786

The energy spectrum of a two-dimensional electron gas placed in a transversal magnetic field $B$ consists of quantized Landau levels. In the absence of disorder, the degeneracy of each Landau level is $N=B A / \phi_{0}$, where $A$ is the area of the sample and $\phi_{0}=h / e$ is the magnetic flux quantum. With disorder, localized states appear at the top and bottom of the broadened Landau level, whereas states in the centre of the Landau level (the critical region) remain delocalized. This single-electron theory adequately explains most aspects of the integer quantum Hall effect $^{1}$. One unnoticed issue is the location of the new states that appear in the Landau level with increasing $B$. Here, we show that they appear predominantly inside the critical region. This situation leads to a 'spectral ordering' of the localized states, which explains the stripes observed in measurements of the local inverse compressibility ${ }^{2,3}$, of two-terminal conductance ${ }^{4}$ and of Hall and longitudinal resistances ${ }^{5}$ without the need to invoke interactions as done in previous work ${ }^{6-8}$.

The spectrum and eigenstates of a disorder-broadened Landau level can be studied with the well-established approach of diagonalizing the single-electron hamiltonian

$$
\mathscr{H}=\left(2 m_{\mathrm{e}}\right)^{-1}[-i \hbar \nabla+e \mathbf{A}(\mathbf{r})]^{2}+V(\mathbf{r}),
$$

where $V(\mathbf{r})$ is the disorder. We choose $\mathbf{A}(\mathbf{r})=(0, B x)$ and apply periodic boundary conditions (PBCs) to a system of area $A=L \times L$. Owing to the PBCs, the degeneracy of each Landau level, $N=B L^{2} / \phi_{0}$, is an integer. Usually, in theoretical studies the magnetic field is kept fixed and the electron density $n_{\mathrm{e}}$ or, equivalently, the filling factor $v=n_{\mathrm{e}} A / N$, is swept by adjusting the Fermi energy $E_{\mathrm{F}}$.

As the experiments mentioned above investigate the behaviour of various quantities in the $\left(n_{\mathrm{e}}, B\right)$ plane, we need to understand how the spectrum changes when $B$ is also tuned. Given the constraint that an integer number of fluxes must penetrate the sample, $B$ can only change in discrete steps of $\phi_{0} / L^{2}$. Therefore, we ask the following question: how do single-electron wavefunctions evolve when one more magnetic flux is inserted?

Let $|i, N\rangle, 1 \leq i \leq N$ be the eigenstates of a spin-polarized Landau level corresponding to a given disorder $V(\mathbf{r})$ and a magnetic field $B=N \phi_{0} / L^{2}$. The states are ordered by their energies $E_{1}<E_{2}<\cdots<E_{N}$ (accidental degeneracies can be lifted with minute changes in $V(\mathbf{r}))$.

To see how the wavefunctions evolve when $B$ increases, we calculate their disorder-averaged overlaps:

$$
\bar{D}_{N}(i, j)=\overline{|\langle i, N \mid j, N+1\rangle|^{2}},
$$

where $1 \leq i \leq N$ and $1 \leq j \leq N+1$ label two eigenstates of the hamiltonian (1) with the same disorder potential but different magnetic fields. The overline indicates a disorder average. In the results presented here we typically average over 1,000 disorder realizations, and show results only for the spin-polarized lowest Landau level. Similar results are expected in higher Landau levels. The disorder potential $V(\mathbf{r})$ is modelled as a sum of many shortrange, randomly placed gaussian scatterers. We show four sets of data, for $L=250,400,500,750 \mathrm{~nm}, N=50,128,200,550$ and therefore $B=1.654 \mathrm{~T}$ for the first three data sets and $2.022 \mathrm{~T}$ for the last data set.

The $N$ by $N+1$ matrix $\bar{D}_{N}(i, j)$ is almost zero everywhere except near its diagonal, as shown in Fig. 1a. Focusing on this region in Fig. $1 b$, we see that $\bar{D}_{N}(i, i)$ decreases from near unity to near zero as $i$ increases from 1 to $N$, whereas $\bar{D}_{N}(i, i+1)$ is almost the mirror image of $\bar{D}_{N}(i, i)$. They intersect at $i / N \approx 1 / 2$, that is, half-filling, where they seem to have a universal value, which is independent of $N$ and $B$. These elements change most rapidly in a region near half-filling, which becomes narrower as $N$ increases. Other matrix elements, for example, $\bar{D}_{N}(i, i-2)$ shown in Fig. 1c, exhibit a very small peak in this narrow region which we identify as the critical region.

As the overlap of equation (2) measures the similarity of eigenstates, Fig. 1 reveals that when $B$ increases by $\delta B=\phi_{0} / L^{2}$, the new state appears predominantly in the centre of the disorderbroadened Landau level. This is not surprising because the extended states can enclose a large area (and thus sufficient of the extra flux) so that the effects of the $\delta B \ll B$ increase are not perturbative. In contrast, for localized states the effect of the extra flux is always perturbatively small. Thus, localized states at the bottom of the Landau level are little affected and keep their spectral ordering leading to large overlaps $\bar{D}_{N}(i, i)$. Localized states at the top of the Landau level also keep their spectral ordering but are shifted upwards by 1 , to account for the new eigenstate created in the critical region. This explains why $\bar{D}_{N}(i, i+1)$ is close to unity there.

This conclusion can also be reached using well-known results for the Hofstadter butterfly. To map into these, we tile the infinite plane with copies of the $L \times L$ system, so that the disorder $V(\mathbf{r})$ becomes periodic with period $L$. The resulting Hofstadter problem has as a magnetic unit cell the $L \times L$ area, and thus it corresponds to $B L^{2} / \phi_{0}=q / p=N$. The eigenstates of hamiltonian (1) are now magnetic Bloch waves $\psi_{i, \mathbf{k}}(\mathbf{r})=\mathrm{e}^{-i \mathbf{k} \cdot \mathbf{r}} \mathcal{u}_{i, \mathbf{k}}(\mathbf{r})$. The integer $i$ labels the $q=N$ magnetic Bloch bands (MBBs) originated from a Landau level. The functions $u_{i, \mathbf{k}}(\mathbf{r})$ satisfy generalized PBCs ${ }^{9}$. In effect, each of the $N$ eigenstates of a Landau level of the finite-size $L \times L$ system 

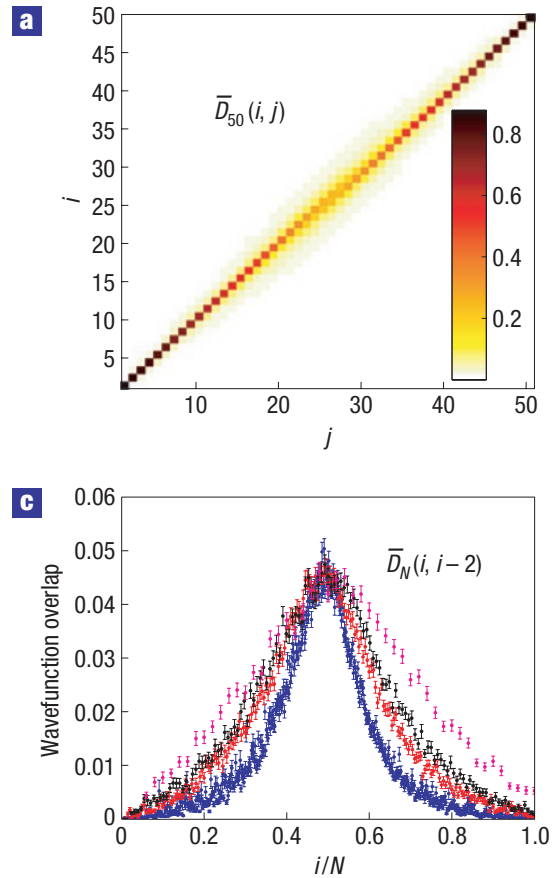

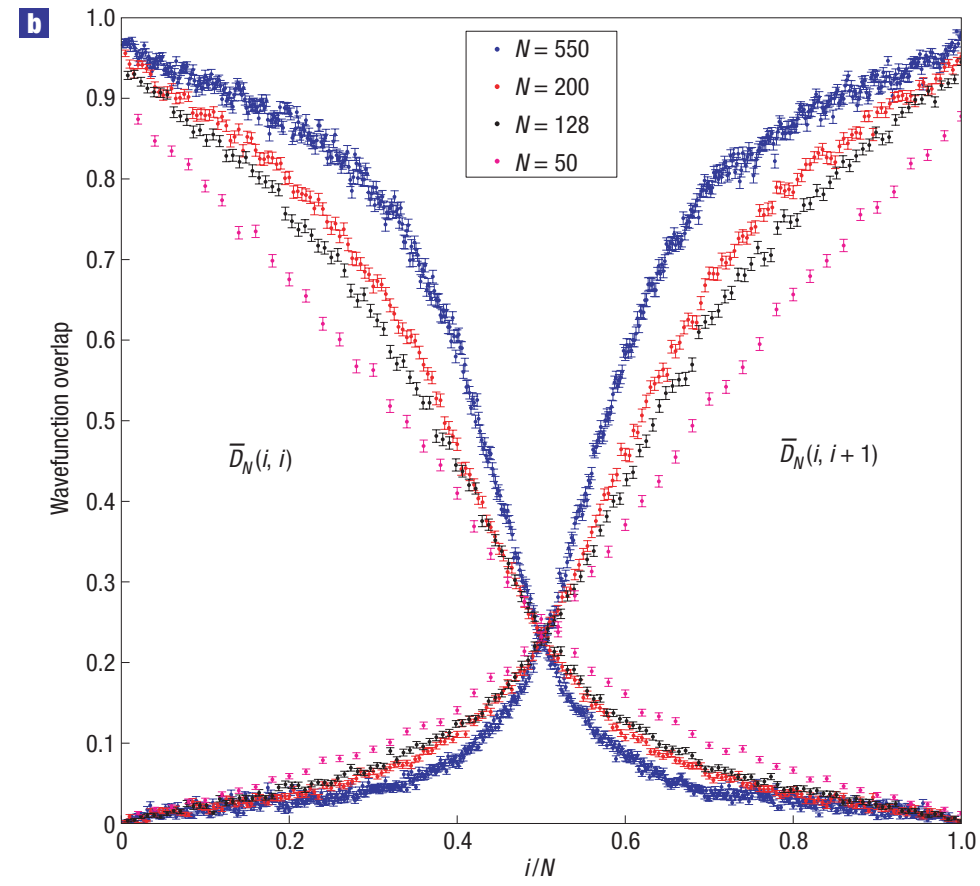

Figure 1 Overlap between eigenstates with an extra magnetic flux quantum. a, Plot of the 50 by 51 overlap matrix $\bar{D}_{50}$, defined in equation (2). Only elements near the diagonal are visible. $\mathbf{b}, \bar{D}_{N}(i, i)$ and $\bar{D}_{N}(i, i+1)$ versus filling factor $i / N$ for four different sizes $N=50,128,200$ and 550 . The transitions sharpen as $N$ increases. c, Typical off-diagonal overlap matrix elements $\bar{D}_{N}(i, i-2)$ versus $i / N$, for the same values of $N$. These are much smaller than the main diagonal elements shown in $\mathbf{b}$. The peak narrows as $N$ increases.

has evolved into an MBB of the Hofstadter problem (the former are the $\mathbf{k}=0$ states of the latter). As a result, we can associate with each eigenstate of the finite-size system the Chern number $\sigma_{i}$ of the corresponding $\mathrm{MBB}^{9} . \sigma_{i}$ is defined for each MBB, because energy bands $E_{i}(\mathbf{k})$ and $E_{i+1}(\mathbf{k})$ may only touch at discrete $\mathbf{k}$ points, and small changes in $V(\mathbf{r})$ remove such degeneracies, as implicitly assumed when $\sigma_{i}$ is calculated in refs 10,11 .

Thouless showed that localized states have zero Chern numbers ${ }^{12}$, which is easy to understand, because localized states are rather insensitive to changes in the boundary conditions used to calculate the Chern number' . This is verified by numerical calculations $^{10,11}$, which find that non-zero Chern numbers appear near the centre of the Landau level, with a distribution in perfect agreement with the scaling theory of the integer quantum Hall effect $^{13-15}$ (IQHE).

When the magnetic field is increased by $\phi_{0} / L^{2}$, that is, $N \rightarrow N+1$, a new MBB must appear in the spectrum generated from each Landau level, and thus one of the original MBBs must split into two. We now argue that only an $\mathrm{MBB}$ with a non-zero Chern number can do this; in other words, the new MBB (new state) appears in the critical region.

A simple proof is obtained by combining Thouless and co-workers' famous proportionality between the Hall conductance of an MBB and its Chern number ${ }^{9}$ and Středa's formula ${ }^{16}$ linking the Hall conductance to the change in the density of states with changing $B$. This gives an expression for the Chern number:

$$
\sigma_{i}=h e^{-1} \partial N_{i}(B) / \partial B
$$

where $N_{i}(B)$ is the density of states in the $i$ th MBB. If this corresponds to a localized state, then $\sigma_{i}=0$ and this MBB cannot be the origin of the new state because its density of states stays unchanged as $B$ varies. The new electronic state in this Landau level must therefore originate from subbands having non-zero Chern numbers.

Further proof for the above result is obtained from the semiclassical theory of Chang and $\mathrm{Niu}^{17,18}$. If $\delta B=\phi_{0} / L^{2}$ supplies the extra flux quantum, the quantization condition of hyperorbits in the MBB reads

$$
\frac{\hbar}{2 e \delta B} \oint_{C_{m}}(\mathbf{k} \times \mathrm{d} \mathbf{k}) \cdot \mathbf{z}+\Gamma_{i}\left(C_{m}\right)=2 \pi(m+1 / 2),
$$

where $m$ is an integer and $\Gamma_{i}\left(C_{m}\right)$ is the contour integral over an effective gauge field $\mathcal{A}_{i}$ along a hyperorbit $C_{m}$. Chang and Niu obtained the entire hierarchical structure of the Hofstadter butterfly by approximating the integral in equation (3) with the area of the magnetic Brillouin zone and replacing $\Gamma_{i}\left(C_{m}\right)$ with the Chern number. If $\sigma_{i}=0$, the localized wavefunction $\psi_{i}(\mathbf{k})$ can be expanded as an absolutely convergent sum of Wannier functions ${ }^{12}$, and the curvature of $\mathcal{A}_{i}$ vanishes identically. Thus, $\Gamma_{i}\left(C_{m}\right)$ indeed vanishes for any localized MBB regardless of the shape of $C_{m}$. In addition, as our magnetic Brillouin zone is $[-\pi / L, \pi / L) \times[-\pi / L, \pi / L)$ by construction, the left-hand side of equation (3) is found to be less than or equal to $\pi$. It follows that $m=0$ is the unique possibility for the MBB of a localized state, that is, such an MBB does not split into multiple MBBs when the magnetic field increases by $\delta B$ (refs 17,18). Note that both arguments are valid only if gaps between neighbouring MBBs remain open as $B$ increases by $\delta B$. As argued, this is expected to be generically true.

A nice illustration of this property is given by the very simple 'disorder' potential $V(\mathbf{r})=t[\cos (2 \pi x / L)+\cos (2 \pi y / L)]$. Of course, this leads to the well-known Hofstadter butterfly 


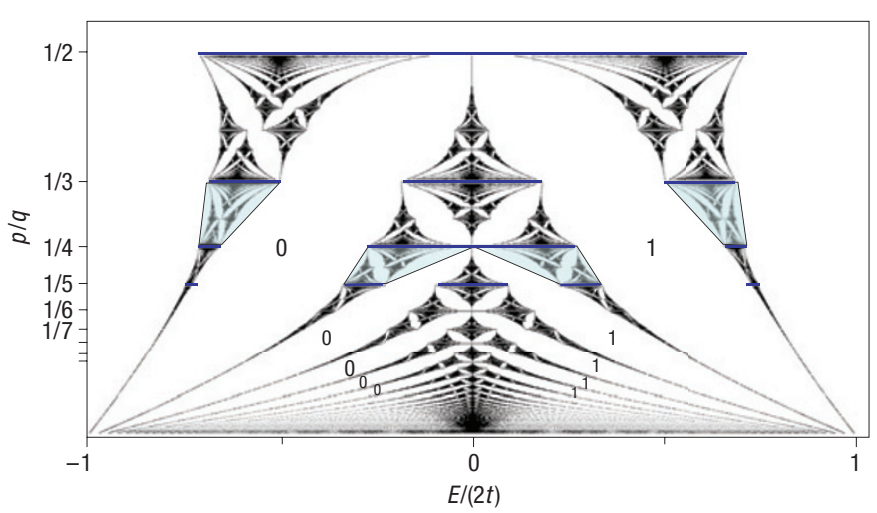

Figure 2 Lower half of a Hofstadter butterfly. The subbands for $p=1, q=N$ are marked by thick blue lines. The Hall conductances in units of $e^{2} / h$ are given for the main gaps, which never close as $B \sim q / p$ increases. The shaded blocks are typical self-similar spectra generated by an MBB when an extra flux is inserted

$(N \rightarrow N+1)$. As $B$ increases, the new spectral weight always appears in the centre of the Landau level.

spectrum, whose lower half is shown in Fig. 2. In accordance with our discussion, we are only interested in magnetic fields $B$ corresponding to $q / p=N$, for large $N$, marked by thick lines in the figure. As expected, there are $N$ MBBs (for even $N$, the two central MBBs just touch). When $N \rightarrow N+1$, a new $\mathrm{MBB}$ is spawned from the central $\mathrm{MBB}(\mathrm{s})$, which is the only one with a non-zero Chern number. Indeed, if $N$ is odd, the central subband evolves into two subbands, whereas if $N$ is even, a new subband grows out in the centre. The outside MBBs have zero Chern numbers and indeed correspond to states localized about the bottom/top of this 'disorder' potential. As argued above, the spectrum of a general disorder potential also has these properties, except that typically there are several MBBs with non-zero Chern numbers, one of which will generate the new state when $B$ increases by one magnetic flux.

To summarize, all of these arguments prove that the new states generated in a disorder-broadened Landau level when the magnetic field increases appear predominantly in the critical region. After a state is expelled to the upper (lower) localized regions as $B$ increases, its order from the top (bottom) of the Landau level remains essentially fixed.

This spectral ordering is the main ingredient needed for understanding the results of recent single-electron transistor (SET) measurements ${ }^{19,20}$ that investigate the charge distribution of localized electronic states in two-dimensional electron systems ${ }^{2,3}$, as well as of measurements of mesoscopic fluctuations of twoterminal conductances ${ }^{4}$ and of Hall and longitudinal resistances ${ }^{5}$. When plotted in the $\left(n_{\mathrm{e}}, B\right)$ plane, the maxima in these quantities are found to track straight lines with certain quantized values for their slopes (see Fig. 3). This suggests that such 'stripes' are an intrinsic aspect of IQHE phenomenology. In fact, SET and transport experiments are strikingly complementary to each other. When their results are put together, as shown schematically in Fig. 3, we get a complete picture of the stripes.

So far it has been unanimously agreed that these stripes are signatures of Coulomb-blockade physics in the localized states ${ }^{6-8}$. We now argue that the main reason for these stripes' appearance is in fact the spectral ordering discussed above, which is a singleelectron effect. Interactions do play a role through screening, as discussed below, but it is very much a secondary one.

We begin our discussion with the SET results that measure the 'local inverse compressibility' $\mathrm{d} \mu / \mathrm{d} n_{\mathrm{e}}$, which is a local d.c. response

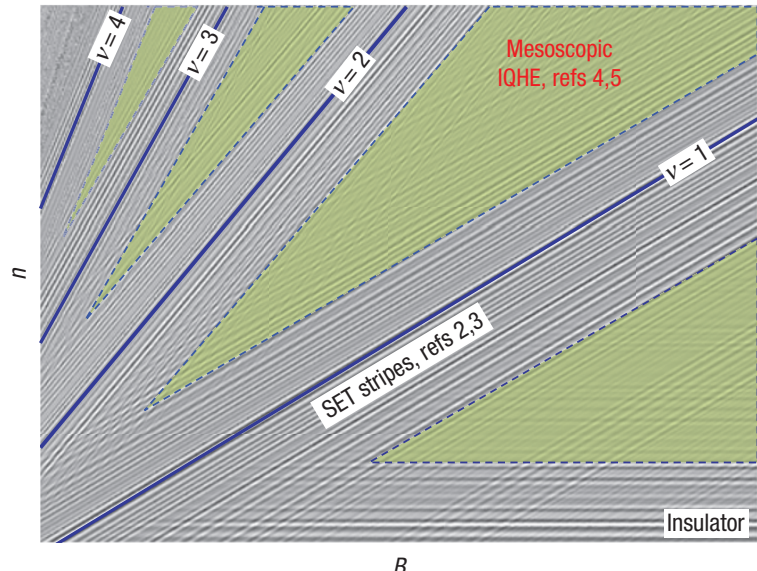

Figure 3 A schematic composite picture (computer-generated) of the stripes observed experimentally in refs $2-\mathbf{5}$. States belonging to the $n$th Landau level are located between the straight lines $n_{\mathrm{e}}=n B / \phi_{0}$ and $n_{\mathrm{e}}=(n+1) B / \phi_{0}$. In the upper half of this Landau level, stripes are found to be parallel to $n_{\mathrm{e}}=(n+1) B / \phi_{0}$, whereas in the lower half, stripes are parallel to $n_{\mathrm{e}}=n B / \phi_{0}$. Near the centre of the Landau level, stripes of both slopes are visible and can cross each other. Refs 2,3 image the stripes close to the Landau level edges, whereas refs 4,5 image the stripes near Landau level centres.

function dominated by localized states located under the SET tip. Consider the evolution of a maximum due to one such localized state, for example one that is found near the bottom of the $n$th Landau level. If the magnetic field is increased by $\delta B=\phi_{0} / L^{2}, n$ new states appear near the centres of the lower $n$ Landau levels (counting from $n=0$ ). As a result, to bring the Fermi level back to this particular state so as to see the same maximum, $n_{\mathrm{e}}$ must be increased by $\delta n_{\mathrm{e}}=n / L^{2}$. Thus, the maximum moves along a line of slope $\delta n_{\mathrm{e}} / \delta B=n / \phi_{0}$. For a state at the top of the $n$th Landau level, however, the density change must be $\delta n_{\mathrm{e}}=(n+1) / L^{2}$, because the spectral position of this state is also shifted upwards by the new state appearing near the centre of the $n$th Landau level itself. Thus, maxima due to these states will have a slope of $(n+1) / \phi_{0}$, precisely as seen in experiments.

It is worth noting that Fig. 4. of ref. 8 shows that even if the Coulomb interaction is turned off, stripes do appear with essentially the right slopes. The authors argue that these are not in agreement with experiment because the region occupied by them increases with $B$, whereas in experiments we see a roughly constant number of maxima, as shown in Fig. 3. Addition of Coulomb interactions fixes this problem, but this is because of screening: their results show that the stronger the interaction, the more effective the screening, the fewer states (maxima) are seen. We therefore argue that Coulomb interactions (screening) have the secondary role of limiting the number of localized states 'visible' to the tip, but the stripes' slopes are determined purely by single-electron physics.

Note that this explanation relies essentially on the fact that localized states tend to keep their spectral order with respect to the top or bottom of the Landau level. Of course, states localized about the same minimum or maximum in the disorder landscape do keep their relative spectral ordering, but it is possible that the energies of states localized in different spatial regions might cross each other as $B$ varies. Such events must be rare, as our simulations in Fig. 1 show; in fact, we find that $\bar{D}_{N}(i, i)$ and $\bar{D}_{N}(i, i+1)$ get closer to 1 in the relevant interval when $N$ increases. However, if such a rare crossing does take place for one of the states under the SET tip, the maximum will shift by $\delta n_{\mathrm{e}}= \pm 1 / L^{2}$ at the $B$ value 
where the crossing occurs, after which the stripe resumes with the correct slope. Such jumps would be impossible to measure.

The stripes in the transport measurements have the same origin. Here, the mesoscopic fluctuations are caused by electronic states that mediate the charge transport across the Hall bar, as shown in refs 21,22 . For example, the fluctuations in the twoterminal conductance measured in ref. 4 are due to Jain-Kivelson tunnelling ${ }^{23}$ through states located in the central region of the sample. To see the same resonance, the Fermi level must be tuned to match the energy of the state mediating the tunnelling, so we expect to see the maxima following lines of quantized slopes in the $\left(n_{\mathrm{e}}, B\right)$ plane for the same reasons given above. However, unlike for SET measurements, the Fermi level is now near the centre of the Landau level, where the transition between quantum Hall plateaux occurs. When an extra $\phi_{0}$ is inserted, there may be either $n$ or $n+1$ new states below the Fermi level. As a result, we expect to see stripes with both slopes. Occasional crossings of stripes are also expected, when the states mediating their tunnelling are more than a coherence length $L_{\phi}$ apart, that is, there is no quantum interference between these two tunnelling events. Finally, these arguments also explain the stripes observed ${ }^{3}$ in the fractional quantum Hall effect ${ }^{24-26}$ (FQHE). It is well known that FQHE can be explained as the IQHE of quasiparticles ${ }^{27}$. Our explanation of the stripes in the IQHE regime is equally applicable to the FQHE regime, with electrons replaced by quasiparticles.

Received 12 July 2007; accepted 16 October 2007; published 2 December 2007.

\section{References}

1. Klitzing, K. v., Dorda, G. \& Pepper, M. New method for high-accuracy determination of the fine-structure constant based on quantized Hall resistance. Phys. Rev. Lett. 45, 494-497 (1980).

2. Ilani, S. et al. The microscopic nature of localization in the quantum Hall effect. Nature $\mathbf{4 2 7}$, 328-332 (2004)

3. Martin, J. et al. Localization of fractionally charged quasi-particles. Science 305, 980-983 (2004)

4. Cobden, D. H., Barnes, C. H. W. \& Ford, C. J. B. Fluctuations and evidence for charging in the quantum Hall effect. Phys. Rev. Lett. 82, 4695-4698 (1999).

5. Jouault, B. et al. Landau levels analysis by using symmetry properties of mesoscopic Hall bars. Phys. Rev. B 76, 161302(R) (2007).
6. Pereira, A. L. C. \& Chalker, J. T. Electrostatic theory for imaging experiments on local charges in quantum Hall systems. Physica E 31, 155-159 (2006).

7. Struck, A. \& Kramer, B. Electron correlations and single-particle physics in the integer quantum Hall effect. Phys. Rev. Lett. 97, 106801 (2006).

8. Sohrmann, C. \& Römer, R. A. Compressibility stripes for mesoscopic quantum Hall samples. New J. Phys. 9, 97-122 (2007).

9. Thouless, D. J. et al. Quantized Hall conductance in a two-dimensional periodic potential. Phys. Rev. Lett. 49, 405-408 (1982).

10. Huo, Y. \& Bhatt, R. N. Current carrying states in the lowest Landau level. Phys. Rev. Lett. 68 1375-1378 (1992).

11. Yang, K. \& Bhatt, R. N. Current-carrying states in a random magnetic field. Phys. Rev. B 55, R1922-R1925 (1997)

12. Thouless, D. J. Wannier functions for magnetic sub-bands. J. Phys. C 17, L325-L327 (1984).

13. Pruisken, A. M. M. Universal singularities in the integral quantum Hall effect. Phys. Rev. Lett. 61, 1297 (1988).

14. Huckestein, B. Scaling theory of the integer quantum Hall effect. Rev. Mod. Phys. 67, 357-396 (1995).

15. Wei, H. P. et al. Experiments on delocalization and university in the integral quantum Hall effect. Phys. Rev. Lett. 61, 1294-1296 (1988).

16. Středa, P. Theory of quantised Hall conductivity in two dimensions. J. Phys. C 15, L717-L721 (1982).

17. Chang, M.-C. \& Niu, Q. Berry phase, hyperorbits, and the Hofstadter spectrum. Phys. Rev. Lett. 75, 1348-1351 (1995).

18. Chang, M.-C. \& Niu, Q. Berry phase, hyperorbits, and the Hofstadter spectrum: Semiclassical dynamics in magnetic Bloch bands. Phys. Rev. B 53, 7010-7023 (1985).

19. Yoo, M. J. et al. Scanning single-electron transistor microscopy: Imaging individual charges. Science 276, 579-582 (1997).

20. Zhitenev, N. B. et al. Imaging of localized electronic states in the quantum Hall regime. Nature 404, $473-476$ (2000).

21. Zhou, C. \& Berciu, M. Resistance fluctuations near integer quantum Hall transitions in mesoscopic samples. Europhys. Lett. 69, 602-608 (2005).

22. Zhou, C. \& Berciu, M. Correlated mesoscopic fluctuations in integer quantum Hall transitions. Phys. Rev. B 72, 085306 (2005).

23. Jain, J. K. \& Kivelson, S. A. Quantum Hall effect in quasi one-dimensional systems: Resistance fluctuations and breakdown. Phys. Rev. Lett. 60, 1542-1545 (1988).

24. Tsui, D. C., Stormer, H. L. \& Gossard, A. C. Two-dimensional magnetotransport in the extreme quantum limit. Phys. Rev. Lett. 48, 1559-1562 (1982).

25. Laughlin, R. B. Anomalous quantum Hall effect: An incompressible quantum fluid with fractionally charged excitations. Phys. Rev. Lett. 50, 1395-1398 (1983).

26. Haldane, F. D. M. Fractional quantization of the Hall effect: A hierarchy of incompressible quantum fluid states. Phys. Rev. Lett. 51, 605-608 (1983).

27. Jain, J. K. Composite-fermion approach for the fractional quantum Hall effect. Phys. Rev. Lett. 63 , 199-202 (1989).

Acknowledgements

We thank B. Jouault and X.-G. Zhang for many stimulating discussions and insightful opinions. This research was carried out at the Center for Nanophase Materials Sciences, sponsored at Oak Ridge National Laboratory by the Division of Scientific User Facilities, US Department of Energy. M.B. acknowledges support from the Sloan Foundation, CIfAR Nanoelectronics and NSERC.

Correspondence and requests for materials should be addressed to C.Z.

Reprints and permission information is available online at http://npg.nature.com/reprintsandpermissions/ 